\title{
COMMENT ON: THE FUTURE IS NEAR: FOCUS ON MYOPIA
}

Singapore Med J 2018; 59(9): 506 https://doi.org/10.11622/smedj.2018111

Dear Sir,

I read with great interest the article 'The future is near: focus on myopia', which was a good piece for the time it was published.(1) The prevalence of myopia is high and has increased across Eastern Asia; nearly 50\% of urban populations are myopic, with a prevalence of between $12.5 \%$ and $90 \% .{ }^{(2)}$ Two popular, efficient myopia control techniques available are orthokeratology (OK) lens treatment and atropine eye drops.

OK treatment was reported to reduce myopia progression by up to $51 \%$. ${ }^{(3)}$ Short-term changes with treatment were reportedly reversible. Central corneal thinning was found to correlate with duration of wear, with decreased density of the basal cell after a fiveyear wear. ${ }^{(4,5)}$ Other long-term effects include a decrease in basal tear secretion and pigment deposition of a corneal iron ring. Low-dose atropine $(0.01 \%-1 \%)$ prescribed nightly was reported to have short-sightedness retardation effects of up to approximately $50 \%{ }^{(3)}$ Lower concentrations of atropine were found to reduce rebound upon discontinuation, photophobia and difficulty with near work.

Myopia is a big concern in Eastern Asia. With the increased influence of myopia control treatments, one may wonder whether there will be any long-term effects. At present, applications differ; for instance, United States Food and Drug Administration approved OK therapy for myopia below -6.00 diopters sphere, ${ }^{(6)}$ and atropine application was introduced at $1 \%$ concentration for myopia control. As all research studies have used a time frame of within five years, there is a need to further investigate the safety of these treatments for the long term.

Yours sincerely,

Jho Yan $\underline{\text { Chia }}^{1,2,3}$

${ }^{1}$ Association of Malaysian Optometrists, Kuala Lumpur, ${ }^{2}$ Columbia Asia Hospital - Cheras, Selangor, ${ }^{3}$ UCSI University, Kuala Lumpur, Malaysia. ptosymposium@gmail.com

\section{REFERENCES}

1. Tan DT. The future is near: focus on myopia. Singapore Med J 2004; 45:451-5.

2. Rudnicka AR, Kapetanakis VV, Wathern AK, et al. Global variations and time trends in the prevalence of childhood myopia, a systematic review and quantitative meta-analysis: implications for aetiology and early prevention. Br J Ophthalmol 2016; 100:882-90.

3. Holden BA, Wilson DA, Jong M, et al. Myopia: a growing global problem with sight-threatening complications. Community Eye Health 2015 ; $28: 35$.

4. Zhong X, Chen X, Xie RZ, et al. Differences between overnight and long-term wear of orthokeratology contact lenses in corneal contour, thickness, and cell density. Cornea 2009; 28:271-9.

5. Chia JY, Ariffin A, Mohidin N, et al. Correlation between duration of orthokeratology lens wear and corneal epithelial and stromal thickness in schoolchildren. Acta Clin Croat 2014; Suppl 2: 9-57.

6. Fadel D. Reshaping Ortho-k. Review of Cornea and Contact Lens 2017. Available at: http://www.reviewofcontactlenses.com/article/reshaping-orthok. Accessed June 13,2018 